\title{
Pharmaceutical Technology, Biopharmaceutics and Drug Delivery
}

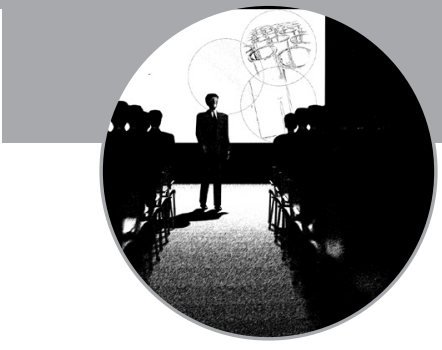

\section{The Korean Society of Pharmaceutical Sciences and Technology 2010 International Conference Jeju Special Self-Governing Providence, Korea, 2-3 December 2010}

The 40th annual international conference of the Korean Society of Pharmaceutical Sciences and Technology on Pharmaceutical Technology, Biopharmaceutics and Drug Delivery was held on 2-3 December 2010 in Jeju Special Self-Governing Providence, Korea, to celebrate its 40th anniversary. A comprehensive review of a wide spectrum of recent topics on pharmaceutical technology, biopharmaceutics and drug delivery was presented. Invited lectures and poster presentations over 2 days were divided into six parallel sessions covering areas such as biotechnology, biopharmaceutics, drug delivery, formulation/manufacture, regulatory science and frontier science. Among these, there were two sessions related to regulatory science and biopharmaceutics that were co-sponsored by the Korea Food and Drug Administration. In fact, this conference provided an opportunity for many investigators to discuss their research, collect new information and to promote the advancement of knowledge in each pharmaceutical area. This conference report summarizes the keynote podium presentations provided by many distinguished speakers, including Gordon L Amidon of the University of Michigan.

The annual international conference of the Korean Society of Pharmaceutical Sciences and Technology (KSPST) provided a dynamic platform for investigators from academia, industry and the public sector to present their scientific work, discuss and compare technology, and expand the scope and range of their research activities. Nearly 300 people from eight different countries including Korea, Japan, China, Vietnam, India, the USA, Australia and New Zealand attended this conference. The running title of this conference was "Current status of pharmaceutical sciences and technology in drug discovery and development". The conference focused mainly on pharmaceutical technologies worldwide and their therapeutic applications. In addition, an attempt was made to strike a balance between conventional and frontier drug-delivery technologies in the programs presented. The conference programs were divided into the following six different sections: pharmaceutical quality and regulations, biotechnology and macromolecular sciences, physical pharmacy and drug delivery, biopharmaceutics, and pharmacokinetics and metabolism, formulation and manufacturing technology, and frontiers in pharmaceutical sciences.

The main themes on the first day were drug delivery, biopharmaceutics and pharmaceutical technology; the conference focused mainly on the following topics:

- Pharmacokinetic/biopharmaceutical considerations for drug-delivery systems;
- Recent advances in pharmaceutical analysis;

- Pulmonary drug delivery of dry powders;

- Nanomaterials/nanocarriers for tumor targeting;

- Molecular imaging;

- Intracellular siRNA delivery.

Beom-Jin Lee (Kangwon National University, Korea) presented findings of on-off pulsedrelease systems according to circadian rhythms for oral drug delivery. His talk focused on the influence of circadian rhythms on the pharmacokinetics and pharmacodynamics of drugs, and explained the basic concept of chronotherapy, which allows for pulsed drug release based on synchronization of drug release with circadian rhythms of the body to achieve both maximum health benefits and minimal harmful effects. He then introduced a novel $\mathrm{pH}$-sensitive pulsatile drug-delivery system; the hydroxylpropylmethylcellulose-based core tablet, which induces on-off pulsed release of a drug-utilizing polyacrylic acid blended with ethylcellulose for $\mathrm{pH}$-sensitivity and fumaric acid as an internal acidifying agent. His results showed dramatic release of bambuterol with an on-off pulsed pattern in vitro.

Kyung-Dall Lee (University of Michigan, MI, USA) provided a comprehensive review on macromolecular therapeutic agents that have the potential to replace or complement conventional

\author{
Yu Seok Youn' \& \\ Beom-Jin Lee ${ }^{\dagger 2}$ \\ 'College of Pharmacy, Pusan National \\ University, Republic of Korea \\ 2Bioavailability Control Laboratory, \\ College of Pharmacy, Kangwon \\ National University, 192-I Hyoja- \\ Dong, Chuncheon-si, Gangwon-do, \\ 200-70I, Republic of Korea \\ ${ }^{\dagger}$ Author for correspondence: \\ Tel.: +82332506919 \\ Fax: +82332423654 \\ E-mail: beomjinlee@gmail.com
}


pharmaceuticals. He spoke about the necessity of appropriate pharmaceutical carriers that are tailored to target and deliver drug treatment to relevant cells and/or subcellular compartments. In this regard, he presented a unique, targeted cytosolic drug-delivery strategy involving nanocarriers for macromolecule drugs using the cell-invasion mechanism of the intracellular bacterium Listeria monocytogenes, to mediate escape from the endocytic compartment into the cytosol of targeted cells using liposomes and polymeric nanoparticles.

Zhonggao Gao (Chinese Academy of Medical Sciences, China) presented several efficient drug-delivery systems based on nanomaterials for cancer-targeting therapy; the significant results included: the targeted delivery of polymeric micelles combined with ultrasound irradiation of tumors; and $\mathrm{pH}$-sensitive polymeric micelles, which were responsive only at the $\mathrm{pH}$ of the tumor, not in the physiological $\mathrm{pH}$ range. In these studies, several functional polymers such as poly(ethylene glycol)- $b$-poly(L-lactide), poly(ethylene glycol)- $b$-poly(caprolactone), and poly(histidine) were used.

Hak-Chan Kim (University of Sydney, Australia) reported on pulmonary drug delivery using dry-powder inhalers. Three major considerations were emphasized: production of the drug powders; characterization and formulation of the powder; and aerosol generation and delivery by the inhaler device in order to succeed in the development of a dry-powder aerosoldelivery system. The presentation also included a review of the fabrication methods such as spray drying and micro-/nano-crystallization used to tailor the production of optimum inhalable dry powders.

Tae Kwan Park (Korea Advanced Institute of Science and Technology, Korea) presented findings on siRNA delivery using nanobiomaterials. A unique design for self-crosslinked and multimerized siRNA (multi-siRNA) via cleavable disulfide linkage, and polyelectrolyte complexes with linear polyethylenimine and multi-siRNA was introduced. The results showed that the multi-siRNA complexes with linear polyethylenimine via disulfide linkages and exhibits enhanced gene-silencing efficiencies through target mRNA-specific RNAi processing without significant immune induction. In addition, findings on aqueous stable 3,4-dihydroxy-L-phenylalamine (DOPA) iron oxide nanoparticles coated with poly(ethylene glycol) and gadolinium chelates, used for dual contrast MRI were presented.
Ick Chan Kwon (Korea Institute of Science and Technology, Korea) reviewed molecular imaging of drug-delivery systems. He introduced a new concept of 'theragnosis' for next-generation cancer treatment that combines early-stage 'diagnosis' and efficient 'therapy', and reviewed in vivo molecular imaging techniques using MRI, positron emission tomography, computed tomography, and near-IR fluorescence imaging to characterize the in vivo behavior of nano-sized drug carriers, including absorption, distribution, metabolism and excretion.

The main themes on the second day of the conference included: pharmaceutical quality, regulatory sciences, and formulation/manufacturing technology; the conference focused on the following major topics:

- Drug-delivery system technologies using the new material siRNA;

- Current status of the biopharmaceutics classification system (BCS);

- Bioequivalence issues;

- Paradigm changes in drug discovery and development;

- Good laboratory practice (GLP) bioanalysis and formulation case study for GLP toxicology;

- New trends in quality systems.

A plenary lecture of this conference given by Gordon L Amidon (University of Michigan, MI, USA) who is one of the founders of the BCS, provided a comprehensive review on biopharmaceutics. A history of biopharmaceutics over the past 50 years was presented by dividing the decades into five different eras. Two major factors brought scientific attention back to biopharmaceutics in the early 1990s: the recognition of the importance of biopharmaceutics based on regulatory standards, international pharmaceutical markets and bioequivalence; and the recognition of the complexity of biological membrane transport in absorption, distribution, metabolism, excretion and toxicology. An intensive review of the BCS based on solubility, drug release and dissolution from the product, and permeability, covered every aspect from drug discovery to bioequivalence issues.

Hiroaki Okada (Tokyo University of Pharmacy and Life Sciences, Japan) lectured on a wide variety of drug delivery system technologies for drug discovery using several interesting biomaterials such as PEGylated antibodies, functional peptides/proteins, oligonucleotides (si/miRNA), and therapeutic 
cells. Poly(lactic-co-glycolic) acid microspheres containing siRNAs targeting VEGF, cFLIP and Raf-1 were reported to exert long-term significant anti-tumor effects with a single intra-tumor injection in a rodent model. The results of sInt- 6 microspheres, in the ASO rat model, showed potential as a long-term neovascularizing agent for siRNA therapy by continuous suppression of target proteins.

Seungpyo Hong (University of Illinois at Chicago, IL, USA) discussed nanohybrid systems with controlled cellular interactions using nanoparticles or liposomes. Three major nanohybrid systems (100-150 nm in diameter) that combine a commonly used polycation, polyethylenimine, with protective outer layers consisting of biodegradable polymeric nanoparticles of either poly(lactide-co-glycolide) or polyethylene-b-poly(lactide-co-glycolide), or PEGylated immunoliposomes, were presented. The results showed the optimum particle size and acceptable loading efficiency of these nanohybrid systems, and suggested that these nanohybrids are promising anticancer agents with regard to cytotoxicity of tumor cells.

The pharmaceutical quality and regulations session was divided into two different streams and was sponsored by the Korea Food and Drug Administration. In the first stream, the invited speakers covered topics such as:

- Current status of bioequivalence studies in Korea (Soo Youn Chung, Korea Food and Drug Administration, Korea);

- Drug development and paradigm changes (Sang M Chung, US FDA);

- Incurred sample reanalysis and variability of reference drugs (Sang Deuk Lee, Bioinfra Co., Ltd, Korea);

- Bioequivalence study designs and statistical considerations (Young-Joo Lee, Kyung Hee University, Korea).
In the other stream, Seung Woo Kang (International Scientific Standards, Ltd, Korea) presented information on bioanalytical method validation for clinical and bioequivalence studies. Hoonjoo Kim (University of Washington, WA USA) provided guidance for new drug applications with regard to the content and qualification of impurities in new drug substances and drug products. Sherry Ku (Anchen Inc., CA, USA) gave a comprehensive lecture on the formulation continuum of discovery animals for GLP toxicology to first-in-human studies. Sang-Hun Seo (Yuhan Corporation, Korea) presented the new trends in quality systems with regard to identity, strength, purity and other quality characteristics to ensure the required levels of safety and efficacy. In addition, guidelines on pharmaceutical development (Q8), quality risk management (Q9) and pharmaceutical quality systems (Q10) were presented.

\section{Summary}

Han-Gon Choi, the chair of the scientific program committee, made the concluding remarks. He summarized the meaning and major outcomes of the 40th anniversary conference of KSPST. In summary, a total of 34 invited lectures were presented together with 172 posters, and nearly 300 participants from eight countries attended the conference.

Financial \& competing interests disclosure This work was supported by a grant from the Korean Health Technology R\&D Project, Ministry of Health and Welfare, Korea. The authors have no other relevant affiliations or financial involvement with any organization or entity with a financial interest in or financial conflict with the subject matter or materials discussed in the manuscript apart from those disclosed.

No writing assistance was utilized in the production of this manuscript. 